\title{
Regulation of Chronic Stress-Induced Changes in Hypothalamic-Pituitary-Adrenal Activity by the Basolateral Amygdala
}

\author{
SEEMA BHATNAGAR, COURTENAY VINING, AND KAI DENSKI \\ Department of Psychology, University of Michigan, Ann Arbor, Michigan 48109, USA
}

\begin{abstract}
Little is known about the role of the basolateral amygdala (BLA) in regulating hypothalamic-pituitary-adrenal (HPA) activity, particularly chronic stress-induced HPA activity. In the current studies, we examined the effects of manipulations of the BLA on HPA responses to the eighth restraint, to novel restraint after repeated cold, or to acute novel restraint alone. Excitotoxic lesions of the BLA, in general, inhibited HPA activity in both acute and chronically stressed animals. To examine the role of the BLA in chronic stress without affecting the response to the first stress, we injected the GABA agonist muscimol to temporarily inactivate the BLA prior to restraint in the same three groups of animals. In contrast to the lesion data, muscimol enhanced the HPA response to acute restraint and to novel restraint after repeated cold, but it did not affect responses to the eighth restraint. These data suggest that the BLA inhibits HPA responses to novel stress but is not important in animals repeatedly exposed to the same stressor. Future studies will focus on the neuroanatomical substrates of BLA's effects on HPA activity including whether inputs from the pPVTh are important.
\end{abstract}

KEYWORDS: stress; hypothalamic-pituitary-adrenal activity; basolateral amygdala; HPA; BLA

\section{INTRODUCTION}

We have previously shown that the posterior paraventricular thalamus (pPVTh) inhibits hypothalamic-pituitary-adrenal (HPA) responses to both novel and familiar stressors in chronically stressed animals without affecting HPA activity in acutely stressed animals. ${ }^{1,2}$ Because the pPVTh does not project directly to the paraventricular hypothalamus, we became interested in its efferent projections and their ability to regulate acute versus chronic stress-induced HPA activity. One set of projections of the pPVTh terminates in the basolateral amygdala (BLA). ${ }^{3}$ However, little is known about the role of the BLA in regulating HPA activity, particularly chronic stress-induced HPA activity. In the present studies, we examined the effects of manipulations of the BLA on HPA responses to acute restraint, to repeated restraint, and to novel restraint after repeated cold. In Experiment 1, we permanently lesioned the

Address for correspondence: Seema Bhatnagar, Department of Psychology, University of Michigan, Ann Arbor, Michigan 48109. Voice: 734-615-3744.

bhatnags@umich.edu

Ann. N.Y. Acad. Sci. 1032: 315-319 (2004). () 2004 New York Academy of Sciences.

doi: 10.1196/annals.1314.050 
BLA and studied HPA responses to the first restraint and to the eighth restraint. BLA lesions inhibited the HPA response to acute restraint, preventing us from examining its regulation of HPA activity under chronic stress conditions. Therefore, in Experiment 2, we injected the GABA agonist muscimol to temporarily inactivate the BLA prior to acute restraint exposure in three groups of rats: rats with no prior stress, rats previously exposed to restraint for 1 week, and rats previously exposed to intermittent cold for 1 week.

\section{METHODS}

All experiments used individually housed adult male Sprague-Dawley rats (225$249 \mathrm{~g}$; Charles-River) and were conducted 2-3 h after lights on. In Experiment 1, BLA lesions were made using NMDA $(0.25 \mu \mathrm{L} ; 20 \mu \mathrm{g} / \mu \mathrm{L}))$ and rats allowed 1 week to recover prior to onset of stress. Rats were exposed to 8 consecutive days of 30min restraint and sampled on day 1 and day 8 . In Experiment 2, guide cannulas were implanted and rats were allowed 1 week to recover prior to onset of stress. Vehicle or musicmol was injected $(0.25 \mu \mathrm{l} ; 1 \mu \mathrm{g} / \mu \mathrm{L})$ bilaterally into the BLA 30 min prior to onset of restraint. Acutely stressed rats were exposed to $30 \mathrm{~min}$ of restraint only and sampled. Repeated restrained rats were exposed to 8 days of 30-min restraint
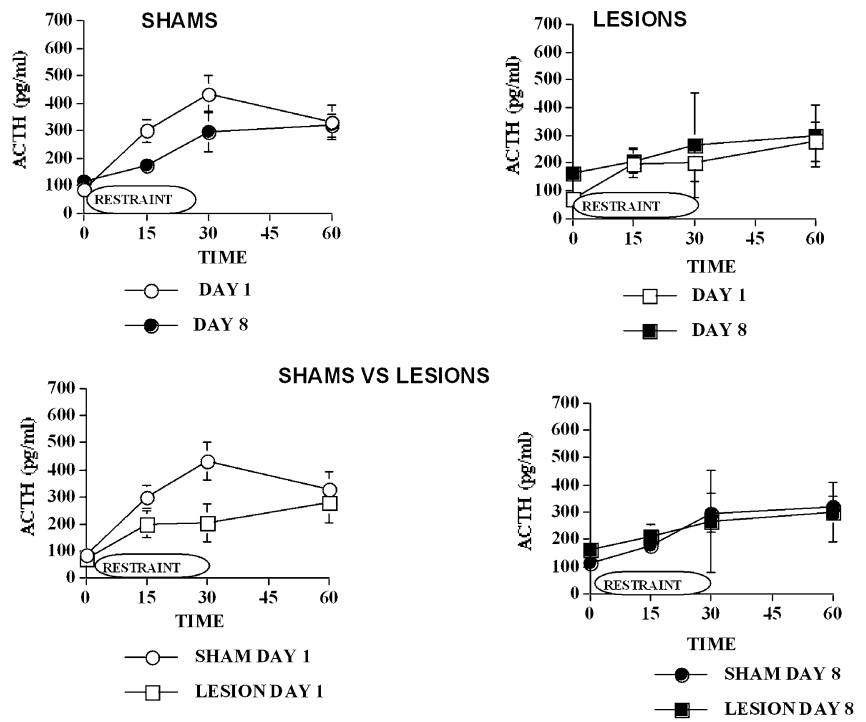

FIGURE 1. ACTH responses to the first and eighth exposure to 30-min restraint are shown in sham- and BLA-lesioned rats. In the top two panels, lower ACTH levels on the eighth exposure to restraint are exhibited by sham-lesioned rats but not by BLA-lesioned rats. These data are re-graphed in the lower two panels and show that on day 1, lesioned rats exhibit lower ACTH responses to restraint than do sham-lesioned rats. By the eighth exposure to restraint, there is no difference between the sham and lesioned rats, because ACTH in sham rats has habituated to the level of the lesioned rats. 
and sampled on day 8 . Repeatedly, cold stressed rats were exposed to $4 \mathrm{~h}$ of cold per day for 7 days and exposed to 30-min novel restraint on day 8 .

\section{RESULTS}

\section{Experiment 1. Effects of BLA Lesions}

Excitotoxic lesions of the BLA, in general, inhibited HPA activity in both acute and chronically stressed animals. The top panels of FIGURES 1 and 2 show that both ACTH and corticosterone, respectively, are lower on day 8 than on day 1 in shamlesioned rats. However, no such differences are observed in BLA-lesioned rats. These data are re-graphed in the bottom panels of both figures to examine the effects of lesions on day 1 or day 8 . These graphs show that even on day 1 , BLA-lesioned
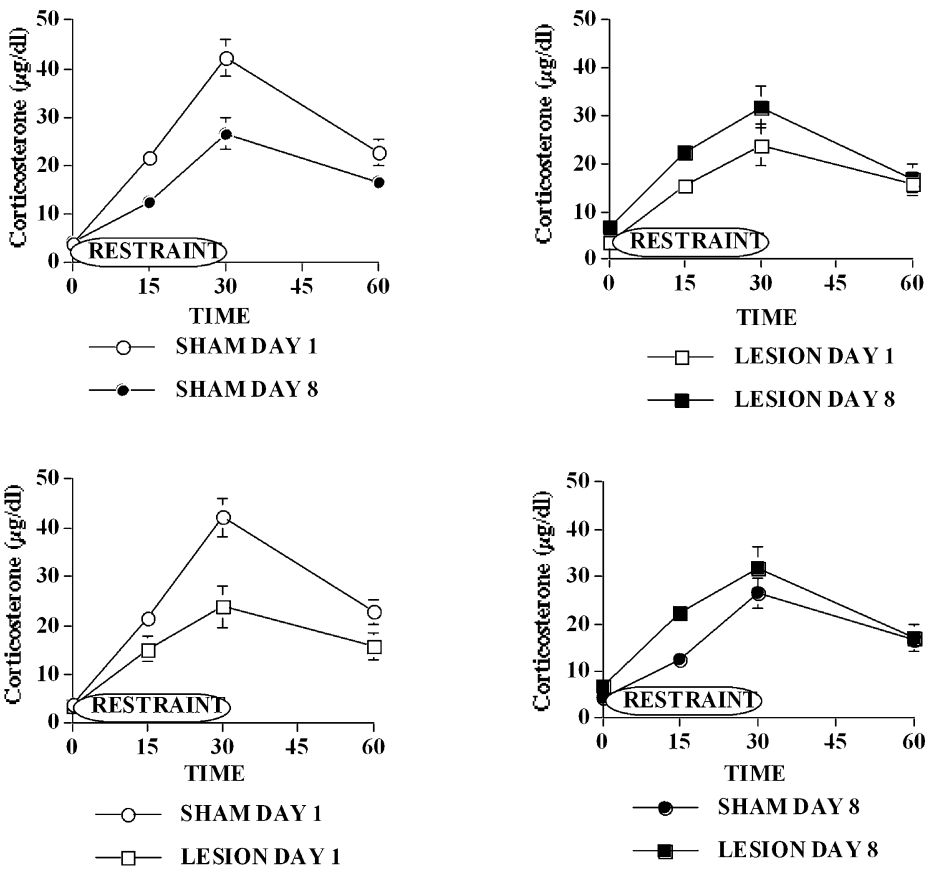

FIGURE 2. Corticosterone responses to the first and eighth exposure to 30-min restraint are shown in sham- and BLA-lesioned rats. As in Figure 1, in the top two panels, lower corticosterone levels on the eighth exposure to restraint are exhibited by sham-lesioned rats but not by BLA-lesioned rats. These data are re-graphed in the lower two panels and show that on day 1 , lesioned rats exhibit lower corticosterone responses to restraint than do sham-lesioned rats. By the eighth exposure to restraint, there is no difference between the sham and lesioned rats, because corticosterone in sham rats has habituated to the level of the lesioned rats. 

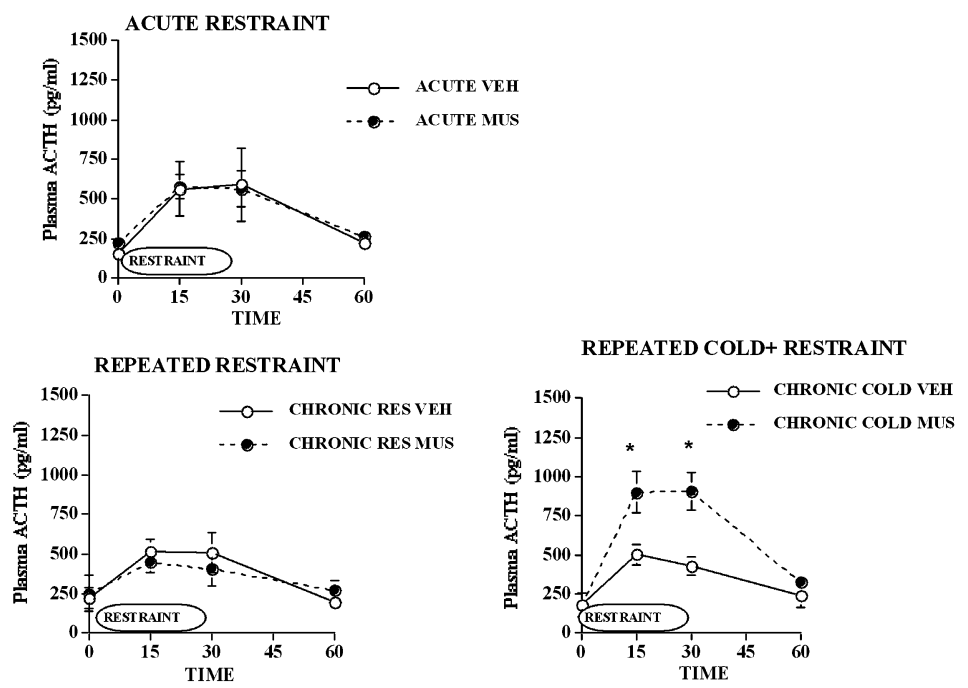

FIGURE 3. ACTH responses to acute restraint, the eighth restraint, or acute novel restraint after chronic cold are shown in rats injected with vehicle or muscimol into the BLA prior to restraint. Muscimol injections into the BLA did not alter ACTH responses to acute restraint or the eighth restraint but increased ACTH responses to restraint in animals previously exposed to 7 days of cold.

rats exhibited lower ACTH and corticosterone responses to restraint than shams. By day 8, BLA-lesioned rats were not different from the sham rats that had habituated to repeated restraint. Together, these graphs show that BLA lesions inhibit responses to acute restraint and that this inhibition continues to be observed on day 8 . As a result, excitotoxic lesions of the BLA do not allow us to discriminate its role in regulating HPA activity in acute versus chronically stressed rats.

\section{Experiment 2. Effects of BLA Inactivation}

In contrast to the lesion data, temporary inactivation of the BLA by local muscimol injections did not affect ACTH responses to acute restraint (FIG. 3). Furthermore, temporary inactivation did not affect ACTH responses to the eighth exposure to restraint. However, muscimol enhanced the HPA response to novel restraint after repeated exposure to a different stressor, cold. Thus, temporary inactivation of the BLA affected HPA activity only in chronically stressed rats exposed to a novel but not a familiar stressor and also did not affect HPA activity in acutely stressed rats.

\section{SUMMARY AND CONCLUSIONS}

Our preliminary data indicate that BLA lesions inhibited HPA responses to acute restraint and, as a consequence, also suppressed repeated stress-induced HPA activ- 
ity. We conclude that excitotoxic lesions of the BLA are not useful in studying how the BLA might regulate HPA activity in chronically stressed rats. Therefore, in the next experiment, we chose to reversibly inactivate the BLA by injecting the GABA agonist muscimol prior to acute restraint exposure in three groups of rats: rats not previously stressed (acute), rats previously exposed to repeated restraint, and rats exposed to repeated cold.

Reversible inactivation of BLA function by local muscimol injections enhanced HPA responses to novel restraint but only in rats previously exposed to a different repeated stressor, cold. Inhibition of BLA function did not alter ACTH responses to restraint if the animals had previous experience with restraint. Finally, muscimol injection did not alter ACTH responses to acute restraint. Therefore, reversible inactivation of BLA function by injection of the GABA agonist muscimol enhanced ACTH responses to novel restraint only if the animals had previous experience with a different stressor. The results of this study suggest that the BLA may play an important role in HPA responsiveness to novelty in organisms with a history of repeated stress.

\section{ACKNOWLEDGMENTS}

This work was supported by National Institute of Mental Health Grant R01 MH067651-01.

\section{REFERENCES}

1. Bhatnagar, S. \& M.F. Dallman. 1998. Neuroanatomical basis for facilitation of hypothalamic-pituitary-adrenal responses to a novel stressor after chronic stress. Neuroscience 84(4): 1025-1039.

2. Bhatnagar, S., R. Huber, N. NowaK, et al. 2002. Lesions of the posterior paraventricular thalamus block habituation of hypothalamic-pituitary-adrenal (HPA) responses to repeated restraint. J. Neuroendocrinol. 14: 403-410.

3. Moga, M.M., R.P. Weiss \& R.Y. Moore. 1995. Efferent projections of the paraventricular thalamic nucleus in the rats. J. Comp. Neurol. 359: 221-238. 